\title{
93. EL ANDROSACION VANDELLII EN EL PIRINEO: ANDROSACETUM PYRENAICAE, NUEVA ASOCIACIÓN DE LOS EXTRAPLOMOS SILÍCEOS
}

\author{
José Luis BENITO ALONSO
}

\begin{abstract}
Androsacion vandelli in the Pyrenees: Androsacetum pyrenaicae, a new community from the siliceous hoverhangs rocks.
\end{abstract}

Palabras clave. Fitosociología; Androsacetalia vandellii, Androsacion vandelli, Vegetación rupícola, Pirineo, Huesca, España, Francia.

Key words. Phytosociology, Androsacetalia vandellii, Androsacion vandelli, rock vegetation, Pyrenees, Huesca, Spain; France.

Mots clés. Phytosociologie, Androsacetalia vandellii, Androsacion vandelli, Végétation rupicole, Pyrénées, Espagne, France.

Este artículo se inscribe en el marco del proyecto de catalogación de la flora y vegetación del Parque Nacional de Ordesa y Monte Perdido (PNOMP) y su zona periférica de protección (Pirineo Aragonés). En esta comunicación avanzamos algunos resultados de la investigación correspondiente a la vegetación rupícola.

El PNOMP destaca por la riqueza de roquedos y pedrizas en su mayor parte calizos, en todos los pisos altitudinales y exposiciones. Son lugares propicios para el establecimiento de un tipo de flora y vegetación muy específico, adaptado a las duras condiciones que imponen dichos ambientes, por lo que son zonas proclive a la aparición de endemismos, muy abundantes como ya hemos comentado en más de una ocasión (Villar \& Benito, 1995).

Uno de estos endemismos es Androsace pyrenaica, planta que habita en los extraplomos silíceos del Pirineo central. Por la singularidad del hábitat que ocupa y lo restringido de su área de distribución, creímos que sería interesante estudiar desde el punto de vista de la fitocenología el comportamiento ecológico de esta planta. Para ello visitamos buena parte de las localidades conocidas en el Pirineo español levantando inventarios. Por otra parte nos pareció oportuno realizar una revisión de las comunidades de los roquedos silíceos del Pirineo descritas hasta el momento con el fin de establecer la relación de esta planta y su fitocenosis con las mismas.

Para la autoría de los nombres de los táxones se ha seguido a Castroviejo et al., 1986-1999, y en su defecto a Bolòs et al., 1990.

\section{ANDROSACION VANDELLII}

Según Meier \& Braun-Blanquet (1934), Braun-Blanquet (1948), Braun-Blanquet et al. (1952) y Focquet (1982), el Androsacion vandellii agrupa las asociaciones de los roquedos silíceos de la alta montaña de los Alpes, Pirineo y Sierra Nevada. En el Pirineo se distribuye desde el piso montano alto (a partir de los $1500 \mathrm{~m}$ de altitud) hasta el límite superior del alpino (hacia los $2800 \mathrm{~m}$ ). La alianza está muy pobremente definida, pues no

Trabajo realizado con la financiación del Instituto de Estudios Altoaragoneses (Diputación de Huesca) y del Departamento de Educación y Cultura del Gobierno de Aragón. 
existen muchas plantas que aparezcan en los tres macizos montañosos citados y que la caractericen. La especie más fiel y que nomina el sintaxon, Androsace vandellii, está ausente en aproximadamente un tercio de las asociaciones descritas. Parece que la unidad se la confiere sobre todo el cortejo de especies acidófilas acompañantes, así como especies de unidades superiores. Esta indefinición se debe a la gran cantidad de endemismos y características locales que hay en cada macizo. Sin embargo, hasta el momento tampoco parece aconsejable la fragmentación de la alianza, pues la atomización sólo complicaría nomenclaturalmente más las cosas.

En el Pirineo hasta el momento se han descrito 10 comunidades incluidas en esta alianza. A continuación daremos una pequeña descripción de cada una de ellas, su distribución y sus diferencias florísticas (tab. 2, fig. 2). De oeste a este de la cordillera se han descrito las siguientes asociaciones:

\section{Cardamino resedifoliae-Primuletum} hirsutae (Turmel 1955) Benito Alonso 1999 [Acta Bot. Malacitana 24: 231]

Lectotypus: Turmel, Mém. Mus. Hist. Nat., sér. B, Bot. 5: 87, tabla 16, invent. 6. 1955

Syn.: Asplenio septentrionalisPrimuletum viscosae Turmel 1955, nom. incorr. et illeg. (Art. 43 y 31); Asplenio septentrionalisPrimuletum latifoliae Rivas Mart., Báscones, T.E. Díaz, Fern. Gonz. \& Loidi 1991 [Itinera. Geobot. 5: 388], nom. incorr. et illeg. (Art. 43 y 31); Asplenio septentrionalis-Primuletum hirsutae Rivas Mart. \& al. 1991 corr. Villar, Sesé \& Ferrández 1997 [Atlas Flora Pirineo Aragonés: LXXII], nom. illeg. (Art. 31); non Asplenio septentrionalis-Primuletum hirsutae (Lüdi 1921) Br.-Bl. in Meier \& Br.-Bl. 1934 [Prodrome des groupements végétaux, fasc. 2. Groupements rupicoles]

Asociación de los roquedos silíceos de los pisos subalpino y alpino del Pirineo central alcanzando el occidental. En la parte española va desde el valle de Canfranc (río Aragón), hasta Chistau-Gistaín (río Cinqueta), penetrando tímidamente en el de Benasque (pero sin cruzar el río Ésera). Por Francia va desde el pico Anie (Pyrénées Atlantiques) hasta el valle de Aure (Hautes Pyrénées). Como especies características podemos citar Asplenium septentrionale y Primula hirsuta. Comparte área de distribución con el Androsacetum pyrenaicae, pero en roquedos no extraplomados, es decir, con inclinación $£$ $90^{\circ}$, por lo que también aparecen plantas más propias de rellanos como Silene rupestris, Sedum brevifolium, Sempervivum montanum, Cerastium alpinum, etc., que no están en la citada asociación. Sobre la nomenclatura de esta comunidad hablamos en Benito (1999b).

\section{Saxifrago cotyledonis-Primuletum hirsutae} Fern. Casas 1970 corr. Benito Alonso 1999

Holotypus: Fernández Casas, Ars. Pharm. 11: 2 , invent. único. 1970

Syn.: Hyperico nummularii-Saxifragetum cotyledonis Rivas Mart. \& al. 1991, nom. superfl.

Comunidad de las grietas de las rocas silíceas, a veces ricas en carbonatos, rezumantes o húmedas buena parte del año, de los pisos montano alto y subalpino del extremo occidental del Pirineo central (alto Valle de Tena). Se caracteriza por la presencia de Saxifraga cotyledon y Primula hirsuta, así como especies del orden y de la clase como Poa nemoralis subsp. glauca o Saxifraga paniculata. Sobre la nomenclatura de esta comunidad véase igualmente Benito (1999b).

\section{Phyteumo hemisphaerici-Cardaminetum resedifoliae Fern. Casas 1970}

Holotypus: Fernández Casas, Ars. Pharm. 11: 295, tabla IX, invent. 1. 1970

Es una asociación que se distribuye por los pisos montano alto y subalpino del Pirineo central meridional, en los macizos de PosetsMaladeta, Castanesa y Turbón (Fernández 


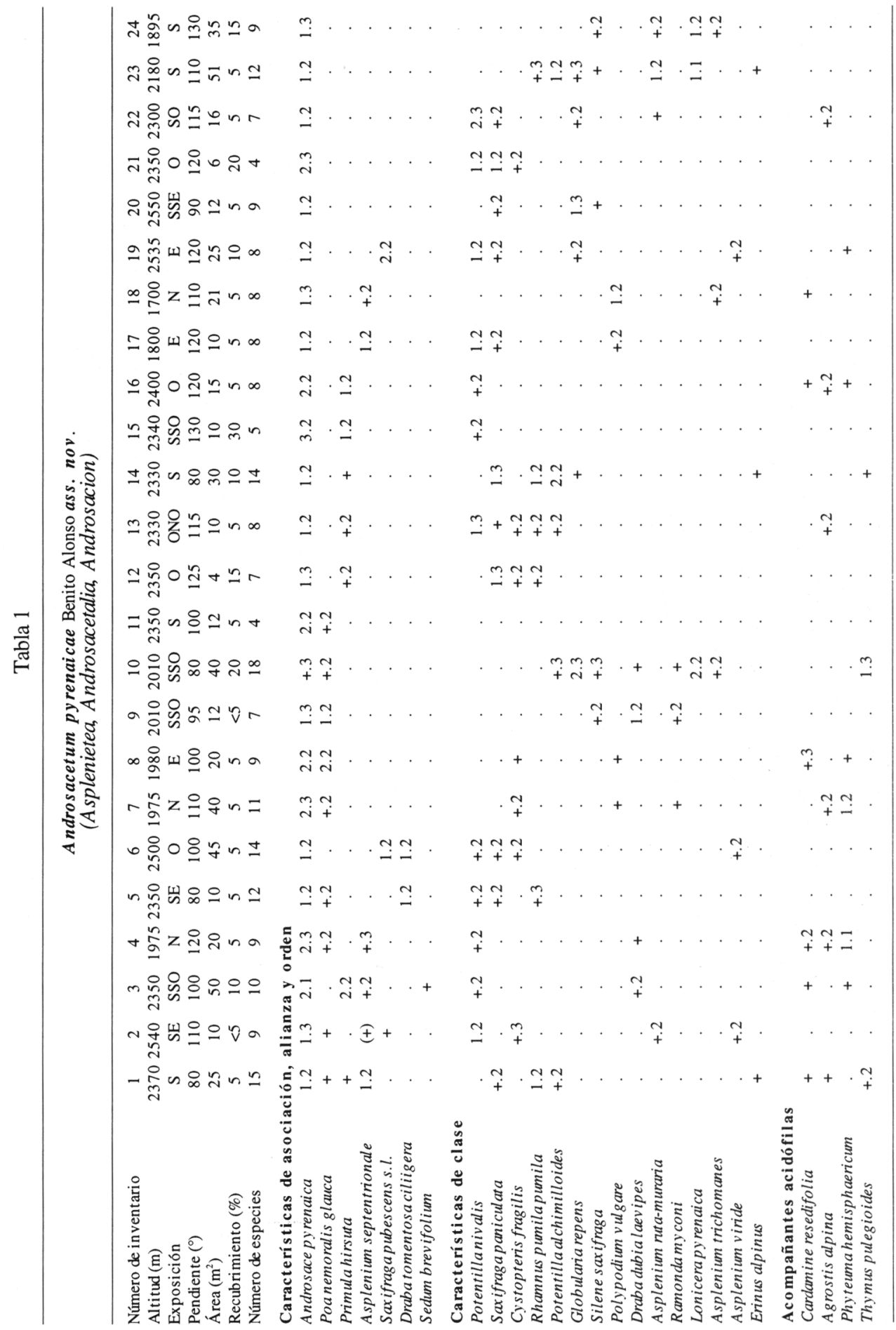




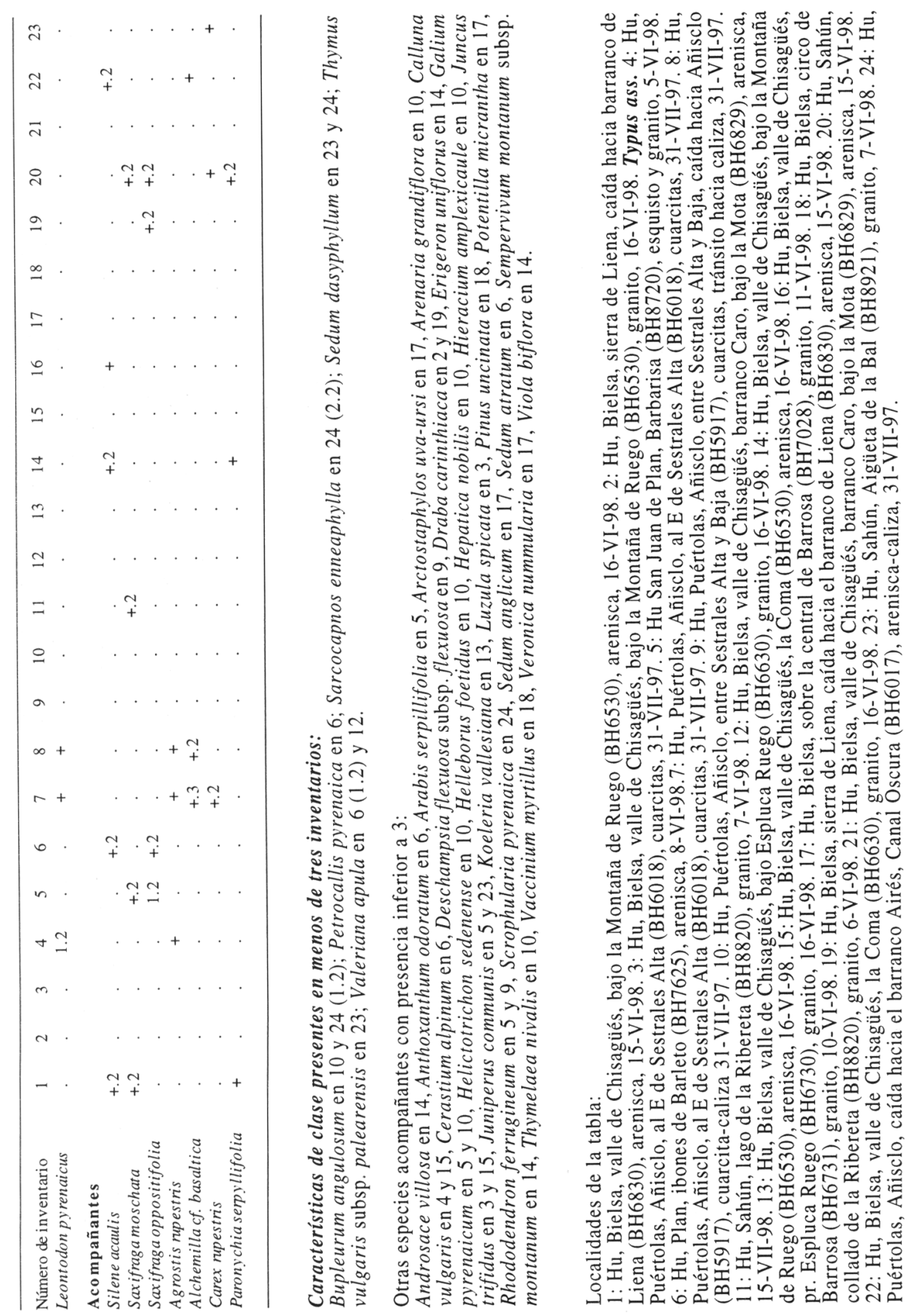




\begin{tabular}{|c|c|c|c|c|c|c|c|c|c|c|c|}
\hline Especie / N. ${ }^{\circ}$ de asociación & 1 & 2 & 3 & 4 & 5 & 6 & 7 & 8 & 9 & 10 & 11 \\
\hline Androsace vandellii & $*$ & . & . & . & $*$ & . & $*$ & $*$ & $*$ & . & $*$ \\
\hline Woodsia alpina & $*$ & . & . & . & . & . & . & . & . & . & . \\
\hline Primula hirsuta & $*$ & * & $*$ & . & . & . & . & . & . & . & . \\
\hline Androsace pyrenaica & + & . & $*$ & . & . & . & + & $\dot{*}$ & . & $\dot{x}$ & $\dot{*}$ \\
\hline Saxifraga pentadactylis & $*$ & . & + & . & . & . & . & $*$ & . & $*$ & $*$ \\
\hline Saxifraga cotyledon & . & * & . & . & . & . & . & . & . & $\cdot$ & $\cdot$ \\
\hline Draba tomentosa subsp. ciliigera & . & . & $*$ & . & . & . & . & . & ; & . & \\
\hline Saxifraga pubescens subsp. pubescens & . & . & * & . & . & . & . & . & $*$ & . & $*$ \\
\hline Saxifraga pubescens subsp. iratiana & . & . & $*$ & . & + & $*$ & . & . & . & . & . \\
\hline Veronica fruticans & . & . & . & * & . & . & . & . & . & . & . \\
\hline Rhodiola rosea & . & . & . & . & $*$ & . & . & . & . & ; & . \\
\hline Saxifraga bryoides & . & . & . & . & $*$ & $*$ & . & . & . & $*$ & . \\
\hline Saxifraga retusa & . & . & . & . & . & + & : & . & . & $*$ & . \\
\hline Saxifraga nervosa & . & . & . & . & . & . & $*$ & ; & . & . & . \\
\hline Draba fladnizensis & . & . & . & . & . & . & . & $*$ & . & . & . \\
\hline Saxifraga aspera & . & . & . & . & . & . & . & $*$ & . & . & . \\
\hline Saxifraga clusii & . & . & . & $*$ & . & . & . & $*$ & : & . & . \\
\hline Artemisia umbelliformis & . & . & . & . & . & . & . & . & $*$ & . & . \\
\hline Poa glauca & . & . & . & . & . & . & . & . & $*$ & $\cdot$ & . \\
\hline Woodsia glabella & . & . & . & . & . & . & . & & $*$ & . & \\
\hline Draba dubia subsp. dubia & . & . & . & . & . & . & . & . & $*$ & . & * \\
\hline Draba subnivalis & . & . & . & . & . & . & . & . & $*$ & . & $*$ \\
\hline Primula latifolia & . & . & . & & . & . & . & . & $*$ & . & $*$ \\
\hline Antirrhinum asarina & . & . & . & . & . & . & . & $\cdot$ & . & $\cdot$ & \\
\hline
\end{tabular}

Tabla 2. Resumen de las especies características de las comunidades pirenaicas del Androsacion vandellii. 1. Cardamino resedifoliae-Primuletum hirsutae. 2. Saxifrago cotyledonis-Primuletum hirsutae. 3. Androsacetum pyrenaicae. 4. Phyteumo hemisphaerici-Cardaminetum resedifoliae. 5. Potentillo nivalisPhyteumetum hemisphaerici. 6. Saxifragetum iratianae. 7. Saxifrago nervosae-Androsacetum vandellii. 8. Saxifrago pentadactylis-Alchemilletum saxatilis. 9. Artemisio gabriellae-Drabetum subnivalis. 10. Saxifragetum retusae. 11. Saxifragetum pubescentis. + = Presencia esporádica.

Casas, 1970: 277) y sierra de Chía (Montserrat, 1989: 114). Tiene como especies más representativas a Phyteuma hemisphaericum, Cardamine resedifolia, Asplenium septentrionale y Veronica fruticans, aunque más bien se caracteriza por la ausencia de especies de unidades superiores, pues sólo Asplenium septentrionale es característica de la alianza. Faltan Primula hirsuta, P. latifolia, Androsace vandellii, A. pyrenaica, Saxifraga pubescens, etc.

El autor de esta comunidad ha descrito también la subasociación saxifragetosum clusii Fern. Casas 1970, que colonizaría los esquistos rezumantes, especialmente ferruginosos, teniendo como especie diferencial Saxifraga clusii.

Holotypus: Fernández Casas, Ars. Pharm.
11: 295, tabla IX, invent. 7. 1970

\section{Potentillo nivalis-Phyteumetum} hemisphaerici Nègre 1968

Lectotypus (designado aquí): Nègre, Portug. Acta Biol.(B), 9(3-4): 223, tabla 4, invent. 12 (n. $\left.{ }^{\circ} 478\right) .1968$

Comunidad de los roquedos del piso alpino de la cara norte del Pirineo central, en la Alta Garona y el extremo occidental del Ariège, si bien en exposiciones de umbría desciende hasta el piso subalpino y en solanas puede ascender a los 2900 m. Las especies características son Phyteuma hemisphaericum, Potentilla nivalis y Androsace vandellii, a las que se añaden Saxifraga bryoides y Thymus nervosus. Sin embargo, se ve mejor caracterizada por la ausencia de plantas de comunidades vecinas 


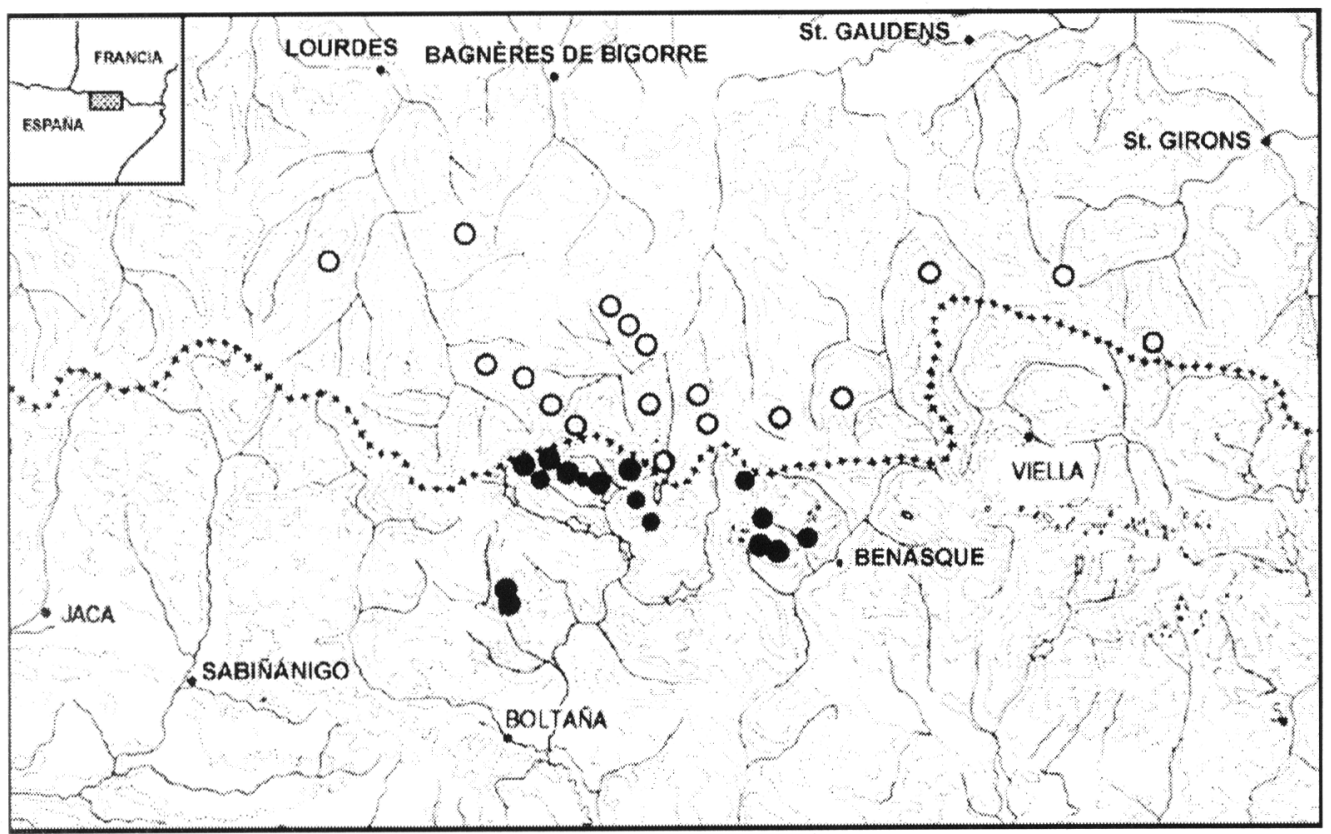

Figura 1. Área de distribución de Androsace pyrenaica Lam. (Primulaceae) en el Pirineo (tomado de Ferrández et al., 1993, modificado y ampliado). O Citas del Pirineo francés según Dupias (1977), ampliado.

como Saxifraga nervosa, Primula hirsuta, Androsace pyrenaica y por la rareza de otras como Poa nemoralis subsp. glauca, Saxifraga pubescens subsp. iratiana o Androsace vandellii. Ha sido citada por Ballesteros \& Canalís (1991: 98) del Besiberri Norte y de Mulleres, pero si aportar ningún inventario de respaldo.

Han sido descritas dos subasociaciones:

La de los pisos subalpino alto y alpino ("sous-association à Thymus nervosus"), que debe ser considerada como la subasociación típica (phyteumetosum hemisphaerici) si atendemos a las indicaciones del protólogo: "L'association [...] occupe les fentes étroites de tous les rochers granitiques ou schisteux, entre $2500 \mathrm{~m}$ et $2750 \mathrm{~m}$ quelle que soit l'exposition, mais au Nord elle desdend jusqu'à 1950 m et au Sud elle peut atteindre 2900".

Y una segunda a menor altitud, rhodioletosum rosae Nègre 1968 nom. mut. prop. ("sous-association à Sedum rhodiola"), en el piso subalpino inferior, caracterizada por la presencia de Rhodiola rosea.

Lectotypus (designado aquí): Nègre, Portug. Acta Biol.(B), 3-4: 223, tabla 4, invent. 2 (n. $\left.{ }^{\circ} 431\right) .1968$.

Proponemos el cambio del nombre original de la subasociación "sedetosum rhodiola", por el de "rhodioletosum rosae" atendiendo al artículo 45 del CNF, según el cual se puede proponer a la Comisión de Nomenclatura la mutación del nombre de un sintaxon cuando el taxon del que deriva no es utilizado por la literatura florística y taxonómica de los últimos años o sólo es citado como sinónimo, tal como ocurre con Sedum rhodiola DC. (cf. Bolòs \& Vigo, 1984; Bolòs et al., 1990; Castroviejo et al., 1997; Dostál, 1989; Greuter et al., 1986; Guinochet \& Vilmorin, 1984; Haeupler \& Schönfelder, 1988; Hämet-Ahti et al., 1986; Hess, 1977; Hejny \& Slavík, 1992; Hultén \& 
Fries, 1986; Jalas et al., 1996; Kerguélen, 1993; Meusel \& Jäger, 1965-1992; Pignatti, 1982; Tutin et al., 1964, 1993).

\section{Saxifragetum iratianae Nègre 1968}

Lectotypus (designado aquí): Nègre, Portug. Acta Biol.(B), 9(3-4): 229, tabla 5, invent. 7 (n. $\left.{ }^{\circ} 372 b\right) .1968$

Syn.: Saxifragetum pubescentis saxifragetosum bryoidis Gruber 1978 p. min. p., [in Gruber, Vég. Pyren. Arieg. Catal. Occ.: 44, tabla. 2, invent. 15. 1978], nom. inval. (Art. 5)

Comunidad pirenaico-central de los pisos alpino y subnival, cuyo límite occidental se encuentra en la divisoria de aguas entre los valles de Benasque y de Oô (Alto Garona). Es una asociación pobre en plantas vasculares y en la que predominan las del género Saxifraga como $S$. pubescens subsp. iratiana, $S$. moschata, $S$. bryoides, con la presencia esporádica de $S$. retusa (Ballesteros \& Canalís, 1991), apareciendo alguna otra planta de ambiente rocoso de altitud como Silene acaulis.

Por otra parte, creemos, al igual que Carrillo \& Ninot (1986), que el inventario 15 de la tabla 2 publicada por Gruber (1978, $u t$ Saxifragetum mixtae), procedente del valle de Arán, se puede adscribir perfectamente a esta asociación.

En la publicación original del Saxifragetum iratianae, Nègre (1968: 228) describió además la subasociación alsinetosum cherleri, en la que hay una presencia casi constante de especies como Minuartia sedoides (= Alsine cherleri), Saxifraga bryoides y Androsace ciliata, así como un cortejo de plantas de Thlaspietalia y Thlaspietea como Cerastium alpinum, Saxifraga oppositifolia, y en menor medida Ranunculus glacialis y Linaria alpina. Los inventarios que corresponden a esta subasociación fueron tomados en lugares con poca pendiente, y no en roquedos, a más de $2900 \mathrm{~m}$ de altitud, en el piso subnival. Por sus semejanzas florísticas y ecológicas, creemos que esta subasociación se corresponde con el Minuartio sedoidisAndrosacetum ciliatae Rivas Martínez 1988, una de las dos comunidades que forman parte de la alianza Androsacion ciliatae, que encuadra a las asociaciones del piso subnival del Pirineo central.

\section{Saxifrago nervosae-Androsacetum vandellii Carrillo \& Ninot 1986}

Holotypus: Carrillo \& Ninot, Fol. Bot. Misc. 5: 100-101, invent. 6. 1986

Syn.: Saxifragetum pubescentis Br.-B1. (1939) 1948 [in Gruber, op. cit.: 44, tabla 2, invent.: 1-7. 1978]; Saxifragetum pubescentis saxifragetosum bryoidis Gruber 1978 p. min. p., [in Gruber, op. cit.: 44, tabla 2, invent. 7 y 19. 1978], nom. inval. (Art. 5)

Se distribuye por los pisos subalpino y alpino, principalmente en la mitad oriental del Pirineo central meridional, desde el Noguera Ribagorzana (Carreras et al., 1993) y el valle de Arán hasta Andorra. Características: Saxifraga nervosa, Androsace vandellii (abundante) y Poa nemoralis subsp. glauca; faltan o escasean Saxifraga pubescens subsp. pubescens, S. pentadactylis y Primula latifolia.

En la mitad occidental de la cadena esta asociación se ve sustituida por el Cardamino resedifoliae-Primuletum hirsutae.

\section{Saxifrago pentadactylis-Alchemilletum} saxatilis Focquet ex Benito Alonso 2000, corr. nom.

Lectotypus (designado aquí): Gruber, Vég. Pyren. Arieg. Catal. Occ., tabla. 2, invent. 19. 1978

Syn.: Saxifrago-Alchemilletum saxatilis Focquet 1982 [Doc. Phytosoc. N.S. 7: 171], nom. inval. (Art. 5); Saxifragetum pubescentis saxifragetosum bryoidis Gruber 1978 p. max. p., [in Gruber, op. cit.: 44, tabla 2, invent. 812, 14, 16, 18, 19. 1978], nom. inval. (Art. 5) Se localiza en la cara norte del Pirineo oriental, en el piso alpino, distribuyéndose por Andorra y el Ariège. Es una comunidad 


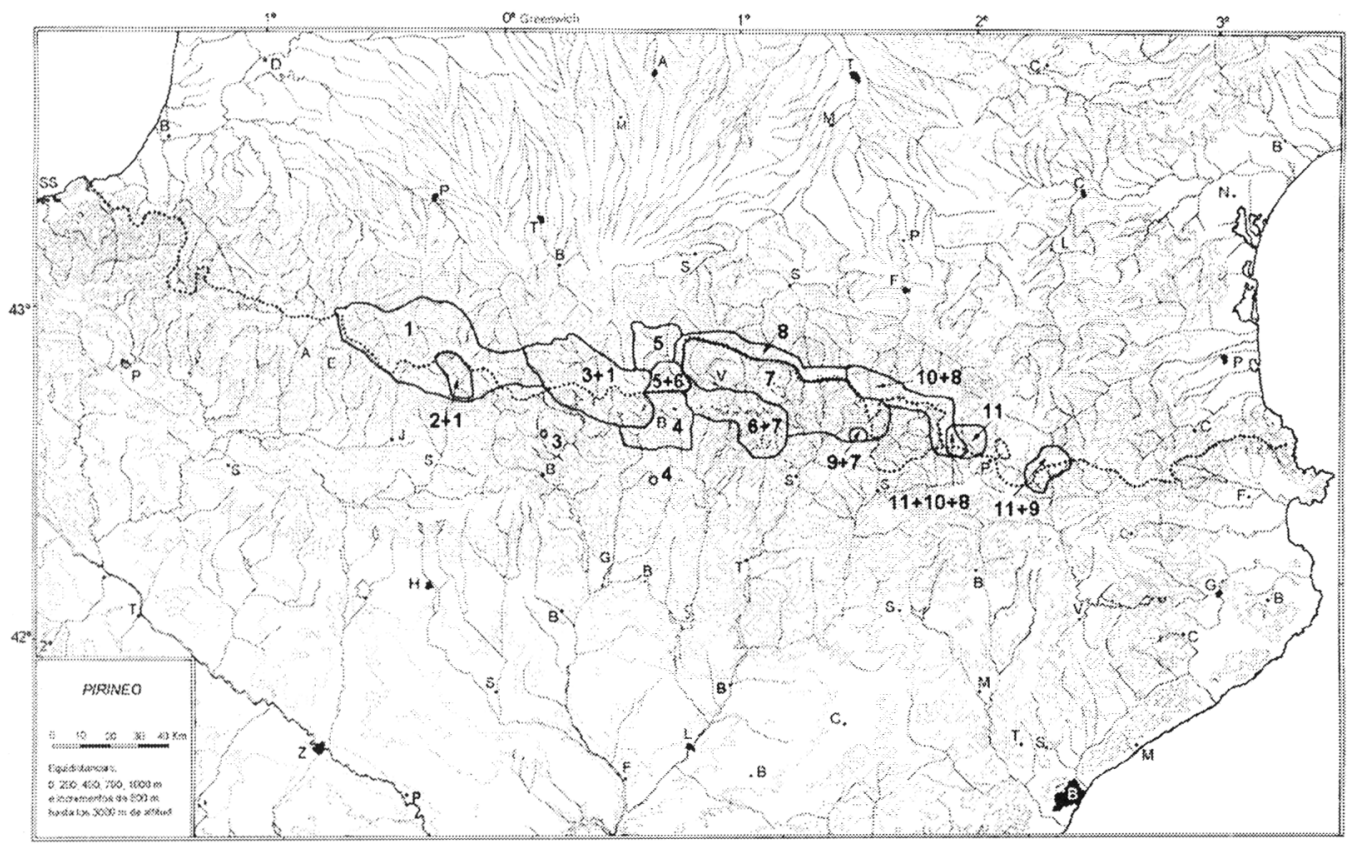

Figura 2. Mapa de distribución de las comunidades del Androsacion vandellii en el Pirineo. 1. Cardamino resedifoliae-Primuletum hirsutae. 2. Saxifrago cotyledonis-Primuletum hirsutae. 3. Androsacetum pyrenaicae. 4. Phyteumo hemisphaerici-Cardaminetum resedifoliae. 5. Potentillo nivalis-Phyteumetum hemisphaerici. 6. Saxifragetum iratianae. 7. Saxifrago nervosae-Androsacetum vandellii. 8. Saxifrago pentadactylis-Alchemilletum saxatilis. 9. Artemisio gabriellae-Drabetum subnivalis. 10. Saxifragetum retusae. 11. Saxifragetum pubescentis.

caracterizada por Saxifraga pentadactylis, $S$. aspera, S. clusii y Draba fladnizensis.

Atendiendo a la recomendación 10D del $\mathrm{CNF}$, se añade el epíteto "pentadactylis" al nombre del sintaxon, con el fin de evitar ulteriores errores de interpretación.

\section{Artemisio gabriellae-Drabetum subnivalis \\ Br.-Bl. 1948}

Lectotypus (designado aquí): BraunBlanquet, Monogr. Estac. Estud. Pir. 9 (Bot. 1): 40 , tabla 3 , invent. 2.1948

Syn.: Potentilletum nivalis Br.-Bl. in Meier \& Br.-Bl. 1934

Comunidad propia del piso alpino de Pirineo axial oriental, preferentemente en solanas. También parece encontrarse en el Pirineo central (pico de Sabollera; Carreras,
1993), aunque muy empobrecida. Características: Artemisia umbelliformis (incl. A. gabriellae, cf. Vallés \& Oliva, 1990), Poa glauca y Woodsia glabella. Suele incluir otras especies rupícolas como Potentilla nivalis (a veces dominante), Saxifraga pubescens subsp. pubescens o Draba subnivalis.

\section{Saxifragetum retusae Gruber 1978}

Lectotypus (designado aquí): Gruber, Vég. Pyren. Arieg. Catal. Occ., tabla. 1, invent. 1. 1978

Comunidad descrita del piso alpino superior del Pirineo centro-oriental septentrional (Ariège, Andorra). Se caracteriza por la presencia de varias saxífragas acidófilas como Saxifraga retusa, $S$. bryoides y $S$. pentadactylis, además de Sedum brevifolium. 
10. Saxifragetum pubescentis $\mathrm{Br} .-\mathrm{Bl} .(1939)$ 1948

Lectotypus (designado aquí): BraunBlanquet, Monogr. Estac. Estud. Pir. 9(Bot. 1): 38-39, tabla 2, invent. 3. 1948

Syn.: Saxifragetum mixtae Br.-Bl. (1939) 1948; Saxifragetum pubescentis saxifragetosum bryoidis Gruber 1978 p. min. p., [in Gruber, op. cit.: 44, tabla 2, invent. 13. 1978], nom. inval. (Art. 5)

Comunidad pirenaico oriental, que se distribuye por los pisos subalpino alto y alpino, desde el valle de Ribes (Vigo, 1996) hasta Andorra y la parte occidental del departamento francés de Pyrénées-Orientales. Características: Saxifraga pubescens subsp. pubescens, S. pentadactylis, Primula latifolia, Draba sp. pl., abundando también Androsace vandellii.

Coincidimos con la interpretación que hacen Carrillo \& Ninot (1986) de la tabla publicada por Gruber (1978), ya que ésta es muy heterogénea y no se ajusta a la asociación descrita por Braun-Blanquet. Por este motivo, no podemos tener en cuenta la subasociación saxifragetosum bryoides Gruber 1978.

Por otra parte, Vigo (1996: 142) describe la subasociación globularietosum repentis, propia de los esquistos más o menos calcáreos, dando como especies diferenciales: Globularia repens, Rhamnus pumilus y Thymelaea dioica.

Holotypus: Vigo, Poblam. Veg. Vall Ribes: 334, tabla 87, invent. 5. 1996

\section{NOVEDADES SINTAXONÓMICAS}

Androsacetum pyrenaicae Benito Alonso, ass. nov.

Holotypus: tabla 1, invent. 3

Combinación florística: como todas las comunidades de extraplomos se caracteriza por una o pocas plantas especialistas en la colonización de ambientes tan singulares y extremos, en este caso Androsace pyrenaica. Suele estar acompañada de un pequeño número de especies características y diferenciales de la alianza Androsacion vandellii y del orden Androsacetalia vandellii como Primula hirsuta (véase mapa en Benito, 1999a), Asplenium septentrionale, Poa nemoralis subsp. glauca $\mathrm{y}$, en ocasiones, Saxifraga pubescens subsp. iratiana, S. pubescens subsp. pubescens o Draba tomentosa subsp. ciliigera. Ahora bien, la mayoría de las veces el cortejo de especies de la clase Asplenietea trichomanis como Potentilla nivalis, P. alchimilloides, Saxifraga paniculata, Cystopteris fragilis, Rhamnus pumila, Globularia repens, etc., es mayor en número que las antes mencionadas.

Ecología: se trata de una comunidad de las paredes extraplomadas y verticales $\left(390^{\circ}\right.$ de inclinación), con sustrato pobre en bases (granito, arenisca, cuarcita, esquisto, etc.) del Pirineo central. Se distribuye por los pisos subalpino y alpino, entre los 1700 y $2600 \mathrm{~m}$ de altitud. El recubrimiento de la vegetación en estos roquedos silíceos suele ser muy bajo, en torno a un $5 \%$, pues o bien la roca presenta pocas fisuras o bien es muy frágil y se fragmenta fácilmente. Sin embargo, nos hemos encontrado con algún ejemplo en el que la cobertura era del $30 \%$ (invent $n .^{\circ} 15$, la Coma, valle de Chisagúés). Allí pudimos contar más de un millar de cojines de Androsace, alguno de los cuales alcanza un tamaño considerable $(15 \mathrm{~cm}$ $\emptyset)$ y porta casi mil flores. Si bien la exposición no condiciona mucho la localización de la comunidad, pues el extraplomo protege de la insolación y la lluvia directas, el Androsacetum pyrenaicae muestra una cierta preferencia por las solanas, como vemos en 13 de los 24 inventarios de la tabla 1.

Fitogeografía: la distribución del Androsacetum pyrenaicae en el Pirineo central de Huesca coincide con la de la especie que le da nombre, pero es probable que esta asociación también se encuentre por todo el área de Androsace pyrenaica en el Pirineo francés (véase figura 1), donde deberá estudiarse. En las mismas localidades pero ya fuera del 
extraplomo, en roquedos menos pendientes encontramos el Cardamino resedifoliaePrimuletum hirsutae.

Sintaxonomía: en la tabla del Saxifragetum mixtae publicada por Gruber (1978: tab. 2, invent. 17), existe un inventario procedente del Ariège en el que aparece Androsace pyrenaica. Sin embargo, no podemos llevarlo a nuestra asociación, pues la presencia de Saxifraga nervosa y S. bryoides (ausentes en el Androsacetum pyrenaicae) permite incluirlo en el Saxifrago nervosae-Androsacetum vandellii algo empobrecido (falta A. vandellii), tal como hacen los autores de esta asociación (Carrillo \& Ninot, 1986: 99).

Conservación: el ambiente en el que se desenvuelve esta comunidad, la alta montaña pirenaica, hace de barrera de la mayor parte de las agresiones que pueda sufrir por parte del hombre. Su núcleo oriental se encuentra enclavado en el seno del Parque Natural de Posets-Maladeta (Ley 6/1998), mientras que la población suroccidental está en el límite del PNOMP. Sin embargo, en la zona donde es más abundante (montaña de Ruego), está prevista la construcción de una estación de esquí alpino, y otra en las cercanías de la población de Punta Suelza. A falta de mayor concreción de los citados proyectos, habrá que tener en cuenta la posibilidad de que el uso de explosivos destruyera alguna población.

\section{APÉNDICE FLORÍSTICO}

Androsace pyrenaica (Primulaceae) es un endemismo del Pirineo central (Ferrández et al., 1993; Castroviejo et al., 1997), tanto español como francés. Es propio de las grietas de las rocas silíceas extraplomadas, fundamentalmente en los pisos subalpino y alpino, aunque puede descender hasta los 1500 $\mathrm{m}$, como en la central de Barrosa (Bielsa) y llegar hasta los $3000 \mathrm{~m}$ en el pico de la Munia. Alcanza el PNOMP en uno de sus pocos afloramientos de cuarcitas, en el macizo de Sestrales (valle de Añisclo-Puértolas); y en su zona periférica, se halla en el macizo de la Munia-Sierra de Liena (Bielsa).

En la actualidad, la distribución de $A$. pyrenaica en el Pirineo español está bien delimitada (fig. 1), sobre todo a raíz de los citados trabajos y al de Sainz Ollero et al. (1996), ciñéndose al Pirineo central de Huesca. El límite occidental lo tiene en Sestrales (valle de Añisclo), mientras que por el este llega hasta el valle de Benasque, aunque no cruza el río Ésera. Existen, no obstante, dos citas antiguas para Lérida, una de Lapeyrouse (1813: 93, ut A. diapensoides), recogida por Bolòs \& Vigo (1996: 53), de la Alta Ribagorza, y otra de Coste (1910: 247) y Coste \& Soulié (1913: 207) del bajo valle de Arán, pero ninguna de ellas ha sido confirmada desde entonces.

Esta especie ha sido incluida en diversos catálogos de especies amenazadas en la categoría en peligro de extinción, como el español (Real Decreto 439/1990), el aragonés (Decreto 49/1995), así como en la normativa europea (Directiva 92/43/CEE, Real Decreto 1997/1995, Directiva 97/62/CE) dentro del Anexo II que incluye a las especies de interés comunitario para cuya protección es necesario designar zonas especiales de conservación. Sin embargo, gracias a las nuevas investigaciones llevadas a cabo por García et al. (1998), han propuesto reconsiderar el status de dicha planta en la legislación, pasándola a la categoría VU: D2 (UICN, 1994).

En el Androsacetum pyrenaicae no encontramos nunca Androsace vandellii. En el Pirineo parece comportarse como una vicariante de $A$. pyrenaica ya que viven en ambientes similares, apareciendo en los extremos del área de distribución de esta última: por el este va desde el Pirineo oriental (Bolòs \& Romo, 1991: 234; Bolòs \& Vigo, 1996: 53) hasta el valle de Benasque, y vuelve a hacer acto de presencia en el macizo de Panticosa (Villar et al., en prensa), dejando un hueco que 
ocupa A. pyrenaica, justamente allí donde la continentalidad climática se hace más acusada.

Al respecto de Saxifraga pubescens debemos decir que hemos encontrado las dos subespecies: la típica en los inventarios 2 y 19 , tomados en el valle de Chisagüés (macizo de la Munia-sierra de Liena), si bien junto a ejemplares que muestran caracteres intermedios; y la subsp. iratiana en el inventario 6. Ya Villar et al. (1997: 295) indicaron la presencia de la subespecie pubescens en el macizo de la Munia (BH63) como límite occidental de distribución.

\section{ESQUEMA SINTAXONÓMICO}

\section{ASPLENIETEA TRICHOMANIS Br.-B1. in Meier \&} Br.-Bl. 1934

+ Androsacetalia vandellii $(=$ multiflorae $) \mathrm{Br}$.Bl. in Meier \& Br.-BI. 1934

* Androsacion vandellii (= multiflorae) $\mathrm{Br}$.B1. in Br.-B1. \& Jenny 1926

Androsacetum pyrenaicae Benito Alonso, ass. nov. [extraplomos silíceos de los pisos subalpino y alpino del Pirineo central]

Artemisio gabriellae-Drabetum subnivalis Br.-Bl. 1948, lectotypus hoc loco (= Potentilletum nivalis $\mathrm{Br}$.-Bi. in Meier \& Br.-Bl. 1934) [roquedos silíceos del piso alpino del Pirineo axial oriental]

Cardamino resedifoliae-Primuletum hirsutae (Turmel 1955) Benito Alonso 1999 (= Asplenio septentrionalis Primuletum viscosae Turmel 1955, nom. incorr. et illeg.; Asplenio septentrionalis-Primuletum latifoliae Rivas Mart. \& al. 1991, nom. incorr. et illeg.; Asplenio septentrionalisPrimuletum hirsutae Rivas Mart. et al. 1991 corr. Villar et al. 1997, nom. illeg.) [roquedos silíceos de los pisos subalpino y alpino del Pirineo central]

Phyteumo hemisphaerici-Cardaminetum resedifoliae Fern. Casas 1970 [roquedos silíceos de los pisos montano alto y subalpino del Pirineo central meridional]

\section{typicum}

saxifragetosum clusii Fern. Casas 1970

Potentillo nivalis-Phyteumetum hemisphaerici Nègre 1968, lectotypus hoc loco [roquedos silíceos del piso alpino del Pirineo central septentrional] phyteumetosum hemisphaerici rhodioletosum rosae Nègre 1968, nom. mut. prop., lectotypus hoc loco

Saxifragetum iratianae Nègre 1968 , lectotypus hoc loco [roquedos silíceos del piso alpino del Pirineo central]

Saxifragetum pubescentis (= mixtae) $\mathrm{Br}$.Bl. (1939) 1948, lectotypus hoc loco [roquedos silíceos de los pisos subalpino y alpino del Pirineo oriental]

typicum

globularietosum repentis Vigo 1996

Saxifragetum retusae Gruber 1978, lectotypus hoc loco [roquedos silíceos del piso alpino superior del Pirineo centro-oriental septentrional]

Saxifrago cotyledonis-Primuletum hirsutae Fern. Casas 1970 corr. Benito Alonso 1999 (= Hyperico nummulariiSaxifragetum cotyledonis Rivas Mart. et al. 1991, nom. superfl.) [roquedos silíceos de los pisos montano alto y subalpino del extremo occidental del Pirineo central (alto Valle de Tena)]

Saxifrago nervosae-Androsacetum vandellii Carrillo \& Ninot 1986 [roquedos silíceos de los pisos subalpino y alpino del extremo oriental del Pirineo central meridional]

Saxifrago pentadactylis-Alchemilletum saxatilis Focquet 1982, corr. nom., lectotypus hoc loco (= Saxifragetum pubescentis saxifragetosum bryoidis Gruber 1978 p. max. p.) [roquedos silíceos del piso alpino del Pirineo oriental septentrional]

THLASPIETEA ROTUNDIFOLII Br.-B1. 1947

+ Thlaspietalia rotundifolii $\mathrm{Br}$ - $\mathrm{Bl}$. in $\mathrm{Br}$ - $-\mathrm{Bl}$. \& Jenny 1926

* Androsacion ciliatae Rivas Mart. 1988

Minuartio sedoidis-Androsacetum ciliatae Rivas Mart. 1988 (= Saxifragetum iratianae alsinetosum 
cherleri Nègre 1968) [crestas silíceas del piso subnival del Pirineo central]

AGRADECIMIENTOS. Quisiera dar las gracias a Daniel Goñi y David Guzmán, con los que compartí unos días de campo, por la cesión desinteresada de alguno de los inventarios publicados en este trabajo. A Luis Villar, José Antonio Sesé y Manuel B. Crespo por aportar ideas que han mejorado el manuscrito original. También a José Vicente Ferrández quien me acompañó a la localidad de Sestrales.

\section{BIBLIOGRAFÍA}

BALLESTEROS, E. \& V. CANALÍS -1991- La vegetació culminal dels massissos de Besiberris i de Mulleres (Pirineus Centrals catalans). Butll. Inst. Cat. Hist. Nat. 59(Sec. Bot. 8): 95-106.

BARKMAN, J.J., J. MORAVEC \& S. RAUSCHERT -1986- Code phytosociological nomenclature, $2^{\text {nd }}$ edition. Vegetatio 67: 145-195.

BENITO ALONSO, J.L. -1999a- Cartografía corológica ibérica. Aportaciones 97 y 98 : Primula hirsuta All. y P. latifolia Lapeyr. Bot. Complutensis 23: 139-142.

BENITO ALONSO, J.L. -1999b- Sobre Primula hirsuta All. y la nomenclatura de dos asociaciones pirenaicas del Androsacion vandellii. Acta Bot. Malacitana 24: 229-233.

BOLÒS, O. \& À.M. ROMO, Eds. - 1991 - Atlas corològic de la flora vascular dels Països Catalans. Volum 2. ORCA: atlas corològic, 2. Institut d'Estudis Catalans. Barcelona.

BOLÒS, O., J. VIGO, R.M. MASALLES \& J.M. NINOT - 1990 - Flora manual dels Països Catalans. 1247 pp. Ed. Pòrtic. Barcelona.

BOLÒS, O. \& J. VIGO -1984-1996- Flora dels Països Catalans, vols. I-3. Editorial Barcino. Barcelona.

BRAUN-BLANQUET, J. -1948- La végétation alpine des Pyrénées Orientales. Monografías de la Estación de Estudios Pirenaicos y del Instituto Español de Edafología, Ecología y Fisiología Vegetal n. ${ }^{\circ}$ 9(Bot. 1). 306 pp. Barcelona.

BRAUN-BLANQUET, J. et al. -1952- Les groupements végétaux de la France méditerranéenne. Montpellier.

CARRERAS, J. -1993- Flora i vegetació de Sant Joan de l'Erm i de la vall de Santa Magdalena (Pirineus Catalans). Institut d'Estudis Ilerdencs. Lérida.

CARRERAS, J., E. CARRILLO, J.M. NINOT, R.M. MASALLES \& J. VIGO -1993- El poblament vegetal de les valls de Barravés i de Castanesa. I-Flora i vegetació. Acta Bot. Barcin. 42: 1-392.

CARRILLO, E. \& J.M. NINOT - 1986- Sobre algunes comunitats rupícoles dels Pirineus catalans. Fol. Bot. Misc. 5: 97-103.

CASTROVIEJO, S. et al., Eds. -1986-1999- Flora iberica, vols. 1-7(1), 8. Real Jardín Botánico, C.S.I.C. Madrid.

COSTE, H. -1910- Catalogue des plantes des Pyrénées. Manuscrito inédito.

COSTE, H. \& J.A. SOULIÉ - 1913- Florule du Val d'Aran. Bull. Géogr. Bot. 23: 91-208.

DECRETO 49/1995 de 28 de marzo, de la Diputación General de Aragón, por el que se regula el Catálogo de Especies Amenazadas de Aragón. Bol. Oficial Aragón 42: 1270-1275.

DIRECTIVA 92/43/CEE del Consejo, de 21 de mayo de 1992, relativa a la conservación de los hábitats naturales y de la fauna y flora silvestres. Diario Oficial Comunidades Europeas L 206: 7-50.

DIRECTIVA 97/62/CE del Consejo, de 27 de octubre de 1997, por la que se adapta al progreso científico y técnico la Directiva 92/43/CEE relativa a la conservación de los hábitats naturales y de la fauna y flora silvestres. Diario Oficial Comunidades Europeas L 305: 42-65.

DOSTÁL, J. -1989- Nová Kvetena CSSR, I. Academia. 767 pp. Praga.

DUPIAS, G. -1977- Atlas floristique du Parc National des Pyrénées occidentales, 1. Tarbes.

FERNÁNDEZ CASAS, J. -1970- Notas fitosociológicas breves, I. Ars. Pharm. 11: 273.

FERRÁNDEZ, J.V., J.A. SESÉ \& L. VILLAR 1993- Androsace pyrenaica Lam. (Primulaceae): planta endémica del Pirineo Central. Corología, ecología y conservación. Lucas Mallada 5: 93-100.

FOCQUET, P. -1982- La végétation des parois siliceuses de la vallée de la Vésubie (Alpes Maritimes - France). Doc. Phytosoc. N.S. 7: 1188.

GARCÍA GONZÁLEZ, M.B., D. GUZMÁN, D. GOÑI \& J. ASCASO -1998- Investigación y 
conservación de las especies de flora pirenaica amenazada en Aragón durante 1997 (LIFEFlora Amenazada). Informe técnico inédito. Diputación General de Aragón.

GREUTER, W., H.M. BURDET \& G. LONG, Eds. -1986- Med-Checklist, 3. Dicotyledones (Convolvulaceae-Labiatae). Conservatoire et Jardin Botanistes de la Ville de Genève \& Botanischer Garden \& Botanisches Museum Berlin-Dahlem. Ginebra.

GRUBER, M. -1978- La végétation des Pyrénées ariégeoises et catalanes occidentales. Université d'Aix-Marseille. Marsella.

GUINOCHET, M. \& R. VILMORIN -1984- Flore de France, fascicule 5. Rosales-Myrtales. CNRS. París.

HAEUPLER, H. \& P. SCHÖNFELDER -1988-Atlas der Farn- und Blütenpflanzen der Bundesrepublik Deutschland. Verlag Eugen Ulmer. 768 pp. Stuttgart.

HÄMET-AHTI, L., J. SUOMINEN, T. ULVINEN, P. UOTILA \& S. VUOKKO -1986Retkeilykasvio. Suomen Luonnonsuojelun Tuky Oy. 598 pp. Helsinki.

HEJNY, S. \& B. SLAVÍK -1992- Kvetena Ceské Republiky, 3. Academia. 544 pp. Praga.

HESS, H.E. -1977- Flora der Schweiz und angrenzender Gebiete. Band 2: Nymphaeaceae bis Primulaceae. Birkhäuser Verlag. Basel \& Stuttgart.

HULTÉN, E. \& M. FRIES -1986- Atlas of north european vascular plants. North of the Tropic of Cancer. Koeltz Scientific Books. 968 pp. Königstein, Alemania.

JALAS, J., J. SUOMINEN, R. LAMPINEN \& A. KURTTO, Eds. -1996- Atlas Florae Europeae, 12. Resedaceae-Platanaceae. The Committee for Mapping the Flora of Europe and Societas Biologica Fennica Vanamo. Helsinki.

KERGUÉLEN, M. -1993-Index synonymique de la flore de France. Muséum National d'Histoire Naturelle, Secrétariat Faune-Flore : XXVIII. 196 pp. París.

LEY 6/1998 de 19 de mayo de Espacios Naturales Protegidos de Aragón. BOA 64, de 3 de junio de 1998: 2508-2520.

MEIER, H. \& J. BRAUN-BLANQUET -1934Prodrome des groupements végétaux, fasc. 2. Groupements rupicoles. Imprimerie Mari-Lant. Montpelier.
MEUSEL, H. \& E.J. JÄGER -1965-1992Vergleichende chorologie der zentraleuropäischen flora. Gustav Fischer Verlag Jena. Stuttgart.

MONTSERRAT MARTí, G. -1989- Las comunidades vegetales rupícolas (clase Asplenietea rupestria) en el Cotiella y la sierra de Chía (Prepirineo central de Huesca). Lucas Mallada 1: 103-132.

NÈGRE, R. -1968- La végétation du bassin de l'One (Pyrénées centrales), I. Portugaliae Acta Biol. 9(3-4): 196-290.

PIGNATTI, S. -1982- Flora d'Italia. Edagricole. 790 pp. Bolonia.

REAL DECRETO 439/1990 de 30 de marzo por el que se regula el Catálogo Nacional de Especies Amenazadas. B.O.E. 82: 9468-9471.

REAL DECRETO 1997/1995 de 7 de diciembre, por el que se establecen medidas para contribuir a garantizar la biodiversidad mediante la conservación de los hábitats naturales y de la fauna y flora silvestres. B.O.E. 310: $37310-$ 37333.

RIVAS MARTÍNEZ, S. -1988- La vegetación del piso alpino superior de los Pirineos. Monogr. Inst. Piren. Ecol. 4: 719-728.

SAINZ OLLERO, H., F. FRANCO MÚGICA \& J. ARIAS TORCAL -1996- Estrategias para la conservación de la Flora amenazada de Aragón. Consejo de Protección de la Naturaleza de Aragón. 221 pp. Zaragoza.

TURMEL, J.-M. -1955- Le pic du Midi d'Ossau. Ecologie et végétation. Mém. Mus. Hist. Nat., sér. B, Bot. 5: 1-208.

TUTIN, T.G. \& al., Eds. -1964 y 1993-Flora Europaea, vol. I. Lycopodiaceae to Platanaceae. Cambridge University Press. Ediciones $1^{\mathrm{a}}$ y $2^{\mathrm{a}}$.

UICN -1994- Categorías de las listas rojas de la UICN, adoptadas por la 40 Reunión del Consejo de la UICN Gland, Suiza [http://www.iucn.org/ themes/ssc/redlists/ssc-rl-s.htm].

VALLÈS XIRAU, J. \& M. OLIVA BRAÑAS - 1990Contribution à la connaissance du groupe d'Artemisia umbelliformis Lam. (Asteraceae) dans les Pyrénées. Bot. Piren.-Cantabr.: 321 330.

VIGO, J. -1996-El poblament vegetal de la Vall de Ribes. Les comunitats vegetals $i$ el paisatge. Institut Cartogràfic de Catalunya. Generalitat 
de Catalunya. 468 pp. Barcelona.

VILLAR, L. \& J.L. BENITO ALONSO -1995-

Esquema de la vegetación del Parque Nacional de Ordesa y Monte Perdido, más su zona periférica. Lucas Mallada 6: 235-273.

VILLAR, L., J.A. SESÉ \& J.V. FERRÁNDEZ 1997- Atlas de la Flora del Pirineo Aragonés, I (Introducción. Lycopodiaceae-Umbelliferae). Consejo de Protección de la Naturaleza de Aragón e Instituto de Estudios Altoaragoneses. XCI+648 pp. Huesca.
VILLAR, L., J.A. SESÉ \& J.V. FERRÁNDEZ -en prensa- Atlas de la Flora del Pirineo Aragonés, II (Ericaceae-Orchidaceae). Consejo de Protección de la Naturaleza de Aragón e Instituto de Estudios Altoaragoneses. Huesca.

Aceptado para su publicación en julio de 2000

Dirección del autor. Instituto Pirenaico de Ecología, C.S.I.C. Apdo. 64. E-22700 Jaca. Huesca. España. C. e.: jlbenito@aragob.es

\title{
94. CONTRIBUCIONES AL CONOCIMIENTO DE LA ALIANZA DESCHAMPSION MEDIAE BR.-BL. IN BR.-BL.ET AL. 1952 EN LAS SIERRAS SUBBÉTICAS DEL SUR DE LA PENÍNSULA IBÉRICA
}

\author{
Juan Antonio TORRES, Antonio GARCÍA-FUENTES, \\ Carlos SALAZAR, Manuel MELENDO y Eusebio CANO
}

Contributions to the knowledge of the Deschampsion mediae Br.-Bl. in Br.-Bl. et al. 1952 alliance in the Subbetic Mountains of the southern Iberian Peninsula.

Palabras clave. Deschampsion mediae, sierras Subbéticas, Andalucía, España.

Key words. Deschampsion mediae, Subbetic Mountains, Andalusia, Spain.

Las características ecológicas de los ambientes donde existen comunidades de la clase Molinio-Arrhenatheretea Tüxen 1937, suelos profundos más o menos húmedos, permiten una gran diversidad florística y de comunidades vegetales. Así, dentro de toda la gama de herbazales y juncales que prosperan en el seno de la clase aparecen diferenciados diversos ordenes (Rivas Martínez et al., 1999) en función de su distribución, grado de hidromorfía e influencia antropozoógena. De entre los órdenes de óptimo mediterráneo, Holoschoenetalia Br.-B1. ex Tchou 1948 incluye dos alianzas: Molinio-Holoschoenion Br.-Bl. ex Tchou 1948 dominada por juncales de Scirpus holoschoenus indiferentes a la naturaleza química del sustrato, y Deschampsion mediae $\mathrm{Br}$--Bl. in $\mathrm{Br}$ - $\mathrm{Bl}$. et al. 1952, representada por pastizales agostantes con dominio de biotipos hemicriptofítico y camefítico, desarrollados sobre sustratos carbonatados, especialmente margas ricas en 